\title{
Fatty acid composition and molecular order of phospholipids from Eurotium chevalieri in response to changes in water activity
}

\author{
Laurence Lesage, ${ }^{1 *}$ Claude Genot, ${ }^{2}$ Eric Record,${ }^{1}$ Christiane Pouliquen ${ }^{1}$ \\ and DANIEL RICHARD-MOLARD ${ }^{1}$ \\ ${ }^{1}$ Laboratoire de Microbiologie et Technologie Céréalières, INRA, BP 527, 44026 Nantes Cédex 03, France \\ ${ }^{2}$ Laboratoire d'Etude des Interactions des Molécules Alimentaires, INRA, BP 527, 44026 Nantes Cédex 03, France
}

(Received 7 December 1992; revised 24 February 1993; accepted 4 March 1993)

\begin{abstract}
The mycelial growth of Eurotium chevalieri was examined at different water activities $\left(a_{w}\right)$ using glycerol as the osmoticum. Growth was optimal at $0.90 a_{w}$ and restricted at $0.995 a_{w}$ highlighting the xerophilic behaviour of $E$. chevalieri. Decreased $a_{\mathrm{w}}$ produced an increase in the proportion of oleic acid $\left(\mathrm{C}_{18: 1}\right)$ at the expense of the proportion of linoleic acid $\left(\mathrm{C}_{18: 2}\right)$ of cellular phospholipids. The degree of unsaturation of phospholipid fatty acids showed a $20 \%$ decrease between 0.995 and $0.80 a_{w}$ of growth. Steady-state fluorescence anisotropy $\left(r_{\mathrm{s}}\right)$ and fluorescence lifetime $(\tau)$ measurements for liposomes prepared from cellular phospholipids of $E$. chevalieri and labelled with DPH (1,6-diphenyl-1,3,5-hexatriene) were made at $25^{\circ} \mathrm{C}$. The lipid order parameter $(S$, describing molecular order) and the rotational correlation time ( $\rho$, describing molecular dynamics) were calculated from $r_{\mathrm{s}}$ and $\tau$ data. Except at $0.995 a_{\mathrm{w}}$, a decrease in $a_{\mathrm{w}}$ was accompanied by increasing $r_{\mathrm{s}}$ and $S$ values, indicating a rigidification of membranes, while $\rho$ values were not significantly different. Plots of order parameters and their first derivatives as a function of temperature exhibited break areas in the temperature range $20-48{ }^{\circ} \mathrm{C}$. These large temperature ranges for lipid transitions could correspond to chain melting of complex lipid systems which made up the liposomes prepared from phospholipids of $E$. chevalieri. However, as $a_{\mathrm{w}}$ decreased, the transition temperatures increased globally, between 0.97 and $0.90 a_{\mathrm{w}}$.
\end{abstract}

\section{Introduction}

The importance of fungi in food microbiology is now widely recognized. Spoilage of food and stored products involves a wide range of fungi that differ greatly in their water requirements (Richard-Molard et al., 1985; Lacey, 1989). Pitt \& Hocking (1977) divided the spoilage of foods by fungi into two broad classes: the spoilage of fresh or perishable foods, and the spoilage of dry or processed and stored foods. In this second class of foods, water activity $\left(a_{\mathrm{w}}\right)$ is the main environmental factor which governs the growth of spoiling fungi and thus the shelf life of foods.

Xerophilic fungi are classified according to their ability to grow at reduced $a_{\mathrm{w}}$, below $0.85 a_{\mathrm{w}}$ according to Pitt (1975). Compared with the published data on osmophilic

\footnotetext{
*Author for correspondence. Present address: Laboratoire de Biotechnologie des Champignons Filamenteux, INRA, Faculté des Sciences de Luminy, Case Postale 929, 13288 Marseille Cedex 09, France. Tel. (33) 914162 85; fax (33) 91416707.
}

Abbreviations: DPH, 1,6-diphenyl-1,3,5-hexatriene; THF, tetrahydrofuran; SUV, small unilamellar vesicles. yeasts, there is still little information on the osmoregulatory mechanisms of xerophilic filamentous fungi (Hocking, 1988). Glycerol accumulation by xerophilic fungi in response to reduced $a_{\mathrm{w}}$ was reported in the xerophilic species Chrysosporium fastidium and xerotolerant species Penicillium chrysogenum (Luard, 1982). Glycerol has since been reported as the major internal osmolyte in four xerophilic fungi, Penicillium janczewskii, Eurotium chevalieri, Wallemia sebi and Polypaecilum pisce and one non-xerophilic species, Penicillium digitatum (Hocking, 1986). As shown by Brown (1978) for yeasts, the non-xerophilic species $\boldsymbol{P}$. digitatum leaked glycerol into the growth medium while xerophilic species such as $P$. janczewskii were able to retain glycerol against a strong concentration gradient. These observations led authors such as Biondi et al. (1991) to conclude that the permeability properties of plasma membrane are involved in the adaptation of fungal species to low $a_{\mathrm{w}}$. Cell membranes constrain the free diffusion of solutes and catalyse specific exchange reactions, which together combine to determine the unique chemical compositions of both the cellular and subcellular compartments. Membrane lipids play a central role in mediating 
membrane functions by acting as physical barriers to diffusion of electrolytes, and are solvents for a variety of constituents and conformational stabilizers of membrane proteins (Hazel \& Williams, 1990).

The active restructuring of membrane lipid composition in response to environmental changes preserves a suitable dynamic state of the bilayer (Singer \& Nicholson, 1972) and restores membrane function (Hazel \& Williams, 1990). Among the numerous parameters which describe the dynamic state of lipids in biological membranes, the concept of membrane fluidity is often used to describe the relative motional freedom of membrane lipids (Russell, 1989). The phospholipid composition, both head-group and fatty acyl chains, and the presence of various sterols are reported as important fluidity-modulating factors in membranes. Generally, higher unsaturations of fatty acids fluidize a membrane and decrease the gel to the liquid-crystalline transition temperature $\left(T_{\mathrm{c}}\right)$ for phospholipids (Russell, 1989). Adler \& Liljenberg (1981) and Hosono (1992) showed that salttolerant yeasts responded to salt stress by decreasing the acyl chain unsaturation of their phospholipids and increasing the sterol to phospholipid ratio, leading to a decrease in membrane fluidity. Evaluation of membrane fluidity is often achieved by measuring the movement of hydrophobic fluorescent probes using fluorescence polarization spectroscopy, the most widely used probe being 1,6-diphenyl-1,3,5-hexatriene (DPH) (Shinitzsky \& Barenholz, 1978). Initially, steady-state fluorescence anisotropy data were interpreted exclusively in terms of microviscosity (associated to membrane fluidity) using the Perrin equation (Shinitzky \& Inbar, 1976). However, it was later shown that these data contain not only information on the dynamics (viscosity) but also on the statics (order) of the membrane, which led to a new form of the Perrin equation including the structural order parameter (Van Blitterswijk et al., 1981). Structural parameters such as the lipid order parameter describe molecular order, whereas motion parameters such as membrane fluidity or microviscosity describe molecular dynamics (Heyn, 1979; Van Ginkel et al., 1989).

This paper describes a study of changes in the fatty acid composition of cellular phospholipids and in the structural and dynamic properties of membrane lipids in relation to the xerophilic behaviour of the filamentous fungus $E$. chevalieri. To determine order and dynamics in membranes, liposomes labelled with a fluorescent probe (DPH) were used and the DPH steady-state fluorescence anisotropy and fluorescence lifetime were measured.

\section{Methods}

Strain. Strain LMTC 2 of Eurotium chevalieri, holomorph form described by Raper \& Fennell (1965), was isolated from mouldy cereal grains and grown on maize grains at $25^{\circ} \mathrm{C}$ for routine maintenance and production of conidia.

Media. The basal medium used was $3 \%(\mathrm{w} / \mathrm{v})$ glucose $/ 1 \%(\mathrm{w} / \mathrm{v})$ yeast extract, $\mathrm{pH} \mathrm{6.4}$. This basal medium was supplemented with glycerol to obtain cultures with different water activities. The appropriate glycerol concentrations were obtained from Chen (1987). The six media were of $a_{\mathrm{w}} 0.995$ (maximal without glycerol), 0.97, 0.93, $0.90,0.86$ and 0.80 . The $a_{\mathrm{w}}$ of these media, measured with an electric hygrometer (Rotronic), did not deviate from the calculated $a_{\mathrm{w}}$ by more than 0.005 .

Cultivation. Conidia from $E$. chevalieri grown on maize grains at $25^{\circ} \mathrm{C}$ for $10 \mathrm{~d}$ were suspended in distilled water containing $0.0033 \%$ Tween 80 and glass beads. After shaking, the suspension of spores was filtered through glass wool and counted with a haemocytometer.

Mycelia were grown in one litre Roux flasks containing $100 \mathrm{ml}$ of each medium inoculated with spore suspension to a final concentration of $10^{4}$ conidia ml ${ }^{-1}$. Cultures were incubated at $25^{\circ} \mathrm{C}$ in nonhermetically sealed containers in which constant relative humidities of $97,93,90,86$ and $80 \%$ were maintained with saturated potassium sulphate, ammonium phosphate, barium chloride, sodium sulphate and ammonium sulphate solutions, respectively (Bizot et al., 1978). For $0.995 a_{w}$, salt solutions were replaced by distilled water. At least six samples were taken at intervals according to the growth rate of $E$. chevalieri at each $a_{\mathrm{w}}$. Incubation was for a maximum of $20 \mathrm{~d}$ at the highest $a_{\mathrm{w}}$ of 0.995 , and $86 \mathrm{~d}$ at the lowest $a_{\mathrm{w}}$ of 0.80 . Experiments were repeated twice.

Growth measurement. Biomass from cultures grown in media of different $a_{\mathrm{w}}$ was washed with 31 distilled water and filtered rapidly under vacuum in a Buchner funnel. Moist mycelia were lyophilized and immediately weighed.

Total lipid extraction. Dry mycelia (from $200-300 \mathrm{ml}$ culture media) were moistened with distilled water $(15 \mathrm{ml})$ before lipid extraction. Lipids were then extracted three times successively with $72.5 \mathrm{ml}$ chloroform/methanol/water $(2: 2: 1 \cdot 8$, by vol.) following the method of Bligh \& Dyer (1959). To remove non-lipid materials, the extracted lipids (evaporated to dryness) were washed with $93.5 \mathrm{ml}$ chloroform/ methanol/water containing $0.77 \% \mathrm{NaCl}(8: 4: 3$, by vol.) according to Folch et al. (1957). The total lipid extract was evaporated to dryness under nitrogen, weighed, redissolved in chloroform and stored at $-20^{\circ} \mathrm{C}$.

Separation of phospholipids. Lipids ( $30 \mathrm{mg}$ ) dissolved in chloroform were separated in different classes on $1 \mathrm{~g}$ Silica Sep-Pak cartridge (Waters) as described by Christie (1982). After elution of neutral lipids with chloroform $(10 \mathrm{ml})$ and elution of glycolipids and sphingolipids with acetone $(40 \mathrm{ml})$, phospholipids were recovered by elution with methanol $(10 \mathrm{ml})$. The phospholipid samples were then evaporated to dryness, resuspended in chloroform and stored at $-20^{\circ} \mathrm{C}$. For quantitative phospholipid determination, phosphorus content was measured by the method of Bartlett (1959). The measured values were multiplied by 25 to give the total phospholipid content.

Fatty acid analysis. Fatty acid methyl esters of phospholipids were prepared by direct transmethylation with $14 \%(\mathrm{w} / \mathrm{v}) \mathrm{BF}_{3}$ in methanol according to the method of Morrison \& Smith (1964). Fatty acid methyl esters were then extracted with hexane and analysed by GLC using a Hewlett Packard 5890 Series II chromatograph equipped with a flame ionization detector and a capillary column $(60 \mathrm{~m} \times 0.32 \mathrm{~mm})$ coated with Carbowax $20 \mathrm{M}$. Oven temperature was $200^{\circ} \mathrm{C}$; detector and injector temperatures were $250^{\circ} \mathrm{C}$. Helium was the carrier gas, at a flow rate of $20 \mathrm{ml} \mathrm{min}^{-1}$ with a split ratio of $1 / 20$. The proportion of each fatty acid methyl ester was calculated from the integrated area of each peak and expressed as a percentage of the total area of all peaks. 
Preparation of DPH-labelled vesicles. Liposomes, prepared from extracted phospholipids as small unilamellar vesicles (SUV), were labelled with the 'fluidity probe' DPH, as described by Lentz (1989). Only phospholipids from mycelia harvested in the early exponential growth phase (i.e. $7,8,10,13,17$ and $55 \mathrm{~d}$ at $a_{\mathrm{w}} 0.995,0.97,0.93,0.90$, 0.86 and 0.80 , respectively) were converted to vesicles. A stock solution of $1 \mathrm{mM}-\mathrm{DPH}$ (Sigma) in tetrahydrofuran (THF) was prepared and stored protected from light at $-20^{\circ} \mathrm{C}$. Seven microlitres of freshly prepared $0.2 \mathrm{~mm}-\mathrm{DPH}$ in THF were added to each aliquot of phospholipid extract (equivalent to $1 \mathrm{mg}$ ). The molar DPH/ phospholipid ratio used was 1:1000. Chloroform and THF were evaporated under a stream of nitrogen for $20 \mathrm{~min}$. To prepare SUV, the lipid residue was hydrated overnight in $7.5 \mathrm{ml}$ deoxygenated $0.2 \mathrm{M}$ phosphate buffer solution ( $\mathrm{pH} 7.5$ ), vortexed to suspend it as multilamellar vesicles and kept at $4{ }^{\circ} \mathrm{C}$ overnight. This suspension was then sonicated ( $40 \%$ duty cycle) for $10 \mathrm{~min}$ under nitrogen in an icebath using a $500 \mathrm{~W}, 2 \mathrm{kHz}$ pulse sonifier (Sons \& Sonics) equipped with a titanium microtip. The sonicated mixture was then centrifuged at $1500 \mathrm{~g}$ for $15 \mathrm{~min}$ at $4{ }^{\circ} \mathrm{C}$. The upper three-quarters of the supernatant was kept for fluorescence experiments, and the pellet was removed. As preliminary experiments had shown no changes in anisotropy values during $24 \mathrm{~h}$ storage of samples at room temperature in the dark, probed vesicles were stored in these conditions and used within $24 \mathrm{~h}$ of their preparation. To avoid light scattering effects in the measurements of fluorescence anisotropy (Lentz et al., 1979), the final concentration of vesicles was about $0.2 \mathrm{mM}$, which corresponded to $A_{352}<0.1$, and quartz cuvettes with pathlength $0.4 \mathrm{~cm}$ in excitation were used.

Fluorescence measurements. Steady-state fluorescence anisotropy and fluorescence lifetime experiments were performed with a SLM $4800 \mathrm{C}$ spectrofluorimeter equipped with a two-chamber temperaturecontrolled cell holder. After excitation of samples at $352 \mathrm{~nm}$ (4 and $0.5 \mathrm{~nm}$ slits for steady-state fluorescence and lifetime experiments, respectively), DPH fluorescence was measured using Oriel $435 \mathrm{~nm}$ long pass filters. A Shott BG18 filter was placed in the excitation pathway to avoid high-order grating scattering. Shutters were closed at all times except during measurements, and gentle stirring of samples was maintained during measurements in order to reduce the possibility of DPH photobleaching. Spectrofluorimeter averaging value was set at 20 for all experiments.

Steady-state fluorescence anisotropy was measured in the T-format with the light modulation and frequency electronics turned off. Excitation light was vertically polarized through a Glan-Thompson polarizer. Emission light was simultaneously analysed using two Polaroid polarizers in vertical $\left(I_{\|}\right)$and horizontal $\left(I_{\perp}\right)$ orientations, respectively. The correcting factor $(G)$ for polarization inherent in the instrument was measured using horizontally polarized excitation light. Control samples of DPH suspension alone and of phospholipids alone were examined, but these readings could be neglected since they contributed less than $3 \%$ to the fluorescence of the complete system.

Steady-state fluorescence anisotropy $\left(r_{\mathrm{s}}\right)$ was calculated using the relationship

$$
\left.r_{\mathrm{s}}=\left(I_{\|}-G I_{\perp}\right) / I_{\|}+2 G I_{\perp}\right)
$$

The $r_{\mathrm{s}}$ values were means of 10 measurements taken on the same sample. Anisotropy measurements were performed from $5-60 \pm 0.2^{\circ} \mathrm{C}$ every $2.5^{\circ} \mathrm{C}$, the actual temperature of the samples being monitored using a PT100 platinum thermocouple. Experiments were done in triplicate. The mean standard deviation of the $r_{\mathrm{s}}$ values was about \pm 0.009 .

The interpretation of anisotropy data in terms of membrane fluidity has been discussed extensively by Lakowicz (1983) and Marangoni (1992). Initially, steady-state anisotropy measurements of membrane probes were converted to microviscosity units with reference to isotropic oils. However, after the development of time-resolved fluorescence anisotropy, more information on the complex motions of the fluorophore in the lipid bilayer became obtainable. Accordingly, $r_{s}$ is resolved into a static part $\left(r_{\infty}\right)$ and a dynamic part $\left(r_{\mathrm{f}}\right)$ as shown in equation 2 .

$$
\begin{gathered}
r_{\mathrm{s}}=r_{\mathrm{f}}+r_{\infty} \\
r_{\mathrm{f}}=\left(r_{0}-r_{\infty}\right) /(1+\tau / \rho)
\end{gathered}
$$

The $r_{\mathrm{f}}$ term is related to the rotational relaxation time of the fluorophore $(\rho)$ and depends on the fluorescence lifetime $(\tau)$ (equation 3 ), while the $r_{\infty}$ part describes the restriction to rotation of the fluorophore and is proportional to the square of the order parameter $(S)$ (equation 4). $r_{0}$ is the maximal fluorescence anisotropy value in the absence of any rotational motion of the fluorophore ( 0.362 for DPH). For DPH, $r_{\infty}$ can be calculated from the steady-state fluorescence anisotropy data $\left(r_{\mathrm{s}}\right)$ using the relationship (equation 5) proposed by Van der Meer et al. (1986) where $m=1 \cdot 71$.

$$
\begin{gathered}
S^{2}=r_{\infty} / r_{0} \\
r_{\infty}=r_{0} r_{\mathrm{s}}^{2} /\left[r_{0} r_{\mathrm{s}}+\left(r_{0}-r_{\mathrm{s}}\right)^{2} / m\right]
\end{gathered}
$$

Thereafter, $S$ can be deduced from the calculated $r_{\infty}$ value and equation (4).

Accordingly, the temperature dependence of the lipid order parameter of DPH was estimated from $r_{\mathrm{s}}$ data obtained at the range of temperatures studied $\left(5-60^{\circ} \mathrm{C}\right)$. Using the Kaleida Graph software program (Macintosh), curves were smoothed and first order derivatives were calculated, smoothed and plotted as a function of temperature to visualize the inflection points, indicative of phase transitions in liposomal membranes. When artificial bilayers of phospholipids or membranes are cooled below a critical temperature, called the transition temperature, they undergo a transition from a fluid state (liquidcrystalline phase) to an ordered and condensed state (gel phase); this temperature is particularly sensitive to phospholipid fatty acid and polar head composition (Brenner, 1984).

Lifetime measurements. Even though $r_{\mathrm{s}}$ for DPH is determined mainly by the static component $r_{\infty}$ in membranes, a strict interpretation of $r_{\mathrm{s}}$ requires fluorescence lifetime measurements on which the dynamic component $r_{\mathrm{f}}$ depends. DPH fluorescence lifetime measurements were done at $25^{\circ} \mathrm{C}$ with unpolarized light (excitation and emission polarizers being taken out), at modulation frequency of $30 \mathrm{MHz}$ (Debye-Sears ultrasonic modulator). The sample and the reference solution ( $1 \mathrm{mg}$ glycogen $\mathrm{ml}^{-1}$; lifetime $=0 \mathrm{~ns}$ ) were placed in the two-chamber turret of the spectrofluorimeter. The results were averaged ( 10 measurements taken on the same sample) and analysed by an interfaced calculator using commercial SLM software based on the routines described by Lakowicz (1983). Each experiment was performed in duplicate. The contribution of lifetime changes to steadystate anisotropy was measured by calculating the rotational correlation time of the fluorophore $(\rho)$ from equations 2,3 and 5 .

$$
\rho=\tau\left(r_{\mathrm{s}}-r_{\infty}\right) /\left(r_{0}-r_{\mathrm{s}}\right)
$$

\section{Results}

\section{Growth}

The effect of $a_{\mathrm{w}}$ and culture age on the mycelial growth of Eurotium chevalieri is shown in Fig. 1. The fungus was able to grow significantly at all $a_{\mathrm{w}}$ tested. As $a_{\mathrm{w}}$ decreased, the lag phase increased, up to $50 \mathrm{~d}$ at $0.80 a_{\mathrm{w}}$. Growth of $E$. chevalieri was drastically restricted at $0.80 a_{\mathrm{w}}$, indicating the minimal $a_{\mathrm{w}}$ requirement of this species. 


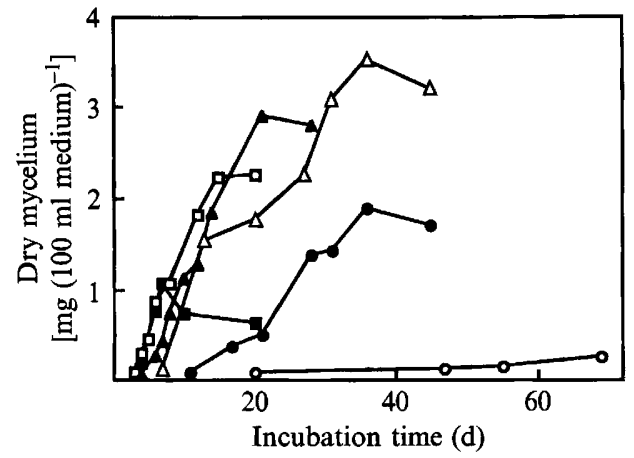

Fig. 1. Effect of $a_{\mathrm{w}}$ and culture age on the mycelial growth of $E$. chevalieri. $\mathbf{\square}, 0.995 a_{\mathrm{w}} ; \square, 0.97 a_{\mathrm{w}} ; \boldsymbol{\Delta}, 0.93 a_{\mathrm{w}} ; \Delta, 0.90 a_{\mathrm{w}} ; \bullet, 0.86 a_{\mathrm{w}}$; O, $0.80 a_{\mathrm{w}}$.

Optimal growth occurred at $0.90 a_{\mathrm{w}}$ while limited growth was observed at $0.995 a_{\mathrm{w}}$.

\section{Fatty acid composition of phospholipids}

Only results obtained from cultures harvested in the early stages of growth (from $7 \mathrm{~d}$ at $0.995 a_{\mathrm{w}}$ to $55 \mathrm{~d}$ at $0.80 a_{\mathrm{w}}$ ) were reported. These cultures yielded mycelia in approximately similar physiological states. Moreover, glycerol accumulation, one of the main osmoregulatory mechanisms of the xerophilic fungi in response to reduced $a_{\mathrm{w}}$, has been described predominantly for early stages of growth (Hocking, 1986). The fatty acid composition of phospholipids from mycelia of $E$. chevalieri grown at $0.995,0.97,0.93,0.90,0.86$ and $0.80 a_{\mathrm{w}}$ is summarized in Table 1 . The major fatty acids were palmitic acid $\left(\mathrm{C}_{16}\right)$, stearic acid $\left(\mathrm{C}_{18}\right)$, oleic acid
$\left(\mathrm{C}_{18: 1}\right)$ and linoleic acid $\left(\mathrm{C}_{18: 2}\right)$. Two odd-chain fatty acids, pentadecanoic acid $\left(\mathrm{C}_{15: 0}\right)$ and margaric acid $\left(\mathrm{C}_{17: 0}\right)$, were detected. As the $a_{\mathrm{w}}$ of the culture medium was reduced, the proportion of $\mathrm{C}_{18: 2}$ decreased substantially, and the proportion of $\mathrm{C}_{18: 1}$ and, less significantly, of $\mathrm{C}_{18: 0}$ increased correspondingly. The changes in distribution of $\mathrm{C}_{18: 2}$ and $\mathrm{C}_{18: 1}$, visualized by the $\mathrm{C}_{18: 2} / \mathrm{C}_{18: 1}$ ratio, were particularly marked at 0.90 , 0.86 and $0.80 a_{\mathrm{w}}$. The degree of unsaturation of phospholipid fatty acids, calculated from the number of double bonds, showed a $20 \%$ decrease between cultures grown at $0.995 a_{\mathrm{w}}$ and those grown at $0.8 a_{\mathrm{w}}$. The relative distributions of other fatty acids were more or less unchanged, and seemed independent of changes in $a_{w}$.

\section{Anisotropy fuorescence and order parameter}

In Table 2, the steady-state fluorescence anisotropy $\left(r_{\mathrm{s}}\right)$ and structural order parameter $(S)$ values at $25^{\circ} \mathrm{C}$ are shown for DPH-labelled liposomes from cellular phospholipids of $7,8,10,13,17$ and 55-d-old $E$. chevalieri grown at $0.995,0.97,0.93,0.90,0.86$ and $0.80 a_{\mathrm{w}}$, respectively. $S$ values were calculated from $r_{\mathrm{s}}$ data according to equations 4 and 5 (see Methods). Except for the sample from mycelium grown at $0.995 a_{\mathrm{w}}$, lower $a_{\mathrm{w}}$ values corresponded to higher $r_{\mathrm{s}}$ and $S$ values, the increase in values being particularly significant at lower $a_{\mathrm{w}}$, i.e. 0.86 and $0.80 a_{\mathrm{w}}$, as shown by standard deviation.

Temperature-dependent changes in $r_{\mathrm{s}}$ values for DPHlabelled liposomal membranes derived from cellular phospholipids of $E$. chevalieri grown at various $a_{\mathrm{w}}$ are presented in Fig. 2. Plots of $r_{\mathrm{s}}$ vs temperature displayed

Table 1. Fatty acid composition of phospholipids from E. chevalieri mycelia harvested in the early stages of growth on media of 0.995, 0.97, 0.93, 0.90,0.86 and $0.80 a_{\mathrm{w}}$

Values (expressed as percentages of total fatty acids) are means \pm SD of two determinations made on mycelia of separate cultures. Values for unsaturation index $\left(\Delta \mathrm{mol}^{-1}\right)$ were calculated according to Kates \& Hagen (1964).

\begin{tabular}{|c|c|c|c|c|c|c|}
\hline \multirow{2}{*}{$\begin{array}{l}\text { Fatty acid } \\
\text { composition }\end{array}$} & \multicolumn{6}{|c|}{$a_{\mathrm{w}}$ (growth time): } \\
\hline & $0.995(7 \mathrm{~d})$ & $0.97(8 \mathrm{~d})$ & $0.93(10 \mathrm{~d})$ & $0.90(13 \mathrm{~d})$ & $0 \cdot 86(17 \mathrm{~d})$ & $0.80(55 \mathrm{~d})$ \\
\hline $\mathrm{C}_{12}$ & $0 \cdot 2 \pm 0 \cdot 1$ & - & $1 \cdot 5 \pm 0 \cdot 4$ & $0 \cdot 5 \pm 0 \cdot 1$ & $0 \cdot 1 \pm 0 \cdot 1$ & $0.9 \pm 0.4$ \\
\hline $\mathrm{C}_{14}$ & $0.1 \pm 0.1$ & $0.2 \pm 0.1$ & $二$ & $0 \cdot 2 \pm 0 \cdot 1$ & $0.2 \pm 0.1$ & $0.6 \pm 0.2$ \\
\hline $\mathrm{C}_{15}$ & 二 & $0 \cdot 5 \pm 0 \cdot 1$ & - & $0.6 \pm 0.2$ & $0.4 \pm 0.1$ & $=$ \\
\hline $\mathrm{C}_{16}$ & $20 \cdot 0 \pm 1 \cdot 1$ & $22 \cdot 8 \pm 1 \cdot 2$ & $20 \cdot 1 \pm 1 \cdot 0$ & $17.6 \pm 0.8$ & $20 \cdot 1 \pm 0.5$ & $23 \cdot 0 \pm 0 \cdot 7$ \\
\hline$C_{16: 1}$ & $0.3 \pm 0.1$ & $1 \cdot 1 \pm 0.5$ & $3 \cdot 3 \pm 1 \cdot 5$ & $0.5 \pm 0.1$ & $0 \cdot 4 \pm 0 \cdot 1$ & $1 \cdot 3 \pm 0 \cdot 4$ \\
\hline $\mathrm{C}_{17}$ & $0 \cdot 6 \pm 0.1$ & $2 \cdot 0 \pm 0.5$ & $1 \cdot 1 \pm 0 \cdot 1$ & $0.6 \pm 0.1$ & $1.2 \pm 0.5$ & $1.3 \pm 0.2$ \\
\hline $\mathrm{C}_{18}$ & $3 \cdot 1 \pm 0 \cdot 3$ & $3.6 \pm 0.5$ & $4.8 \pm 0.2$ & $4.7 \pm 0.3$ & $8 \cdot 0 \pm 1 \cdot 0$ & $4.0 \pm 0.5$ \\
\hline$C_{18.1}$ & $3.3 \pm 0.2$ & $5.7 \pm 0.5$ & $7 \cdot 9 \pm 0 \cdot 1$ & $18.3 \pm 0.8$ & $18.6 \pm 0.7$ & $22.6 \pm 0.5$ \\
\hline$C_{18: 2}$ & $72 \cdot 3 \pm 1 \cdot 5$ & $63 \cdot 9 \pm 1 \cdot 1$ & $61 \cdot 0 \pm 0.5$ & $57.0 \pm 0.7$ & $50 \cdot 9 \pm 0 \cdot 2$ & $45 \cdot 3 \pm 0.2$ \\
\hline$C_{18: 3}$ & $0.1 \pm 0.1$ & $0 \cdot 2 \pm 0.1$ & $0 \cdot 3 \pm 0 \cdot 1$ & 二 & $0.1 \pm 0.1$ & 二 \\
\hline$\Delta \mathrm{mol}^{-1}$ & $1 \cdot 48$ & $1 \cdot 35$ & 1.33 & $1 \cdot 34$ & $1 \cdot 21$ & $1 \cdot 15$ \\
\hline $\mathrm{C}_{18: 2} / \mathrm{C}_{18: 1}$ & 21.9 & $11 \cdot 2$ & $7 \cdot 7$ & $3 \cdot 1$ & $2 \cdot 7$ & $2 \cdot 0$ \\
\hline
\end{tabular}


Table 2. DPH steady-state fluorescence anisotropy $\left(r_{\mathrm{s}}\right)$, order parameter $(S)$, lifetime $(\tau)$ and rotational correlation time $(\rho)$ of liposomes from phospholipids of $E$. chevalieri grown at various $a_{\mathrm{w}}$

Values for $r_{\mathrm{s}}$ and $\tau$ are means \pm SD of at least two separate experiments. The measurements were taken at $25^{\circ} \mathrm{C}$. $S$ and $\rho$ values were calculated as described in Methods.

\begin{tabular}{ccccc}
\hline \hline$a_{\mathrm{w}}$ (growth time) & $r_{\mathrm{s}}$ & $S$ & $\tau(\mathrm{ns})$ & $\rho$ (ns) \\
\hline $0.995(7 \mathrm{~d})$ & $0.1427 \pm 0.0011$ & $0.5051 \pm 0.0036$ & $3.581 \pm 0.151$ & $0.822 \pm 0.053$ \\
$0.970(8 \mathrm{~d})$ & $0.0946 \pm 0.0010$ & $0.3427 \pm 0.0033$ & $3.989 \pm 0.191$ & $0.777 \pm 0.061$ \\
$0.930(10 \mathrm{~d})$ & $0.0955 \pm 0.0009$ & $0.3462 \pm 0.0030$ & $3.692 \pm 0.200$ & $0.722 \pm 0.059$ \\
$0.900(13 \mathrm{~d})$ & $0.1033 \pm 0.0007$ & $0.3734 \pm 0.0023$ & $3.374 \pm 0.180$ & $0.689 \pm 0.050$ \\
$0.860(17 \mathrm{~d})$ & $0.1570 \pm 0.0009$ & $0.5562 \pm 0.0030$ & $3.207 \pm 0.060$ & $0.704 \pm 0.025$ \\
$0.800(55 \mathrm{~d})$ & $0.1823 \pm 0.0011$ & $0.6257 \pm 0.0036$ & $\mathrm{ND}$ & $\mathrm{ND}$ \\
\hline \hline
\end{tabular}

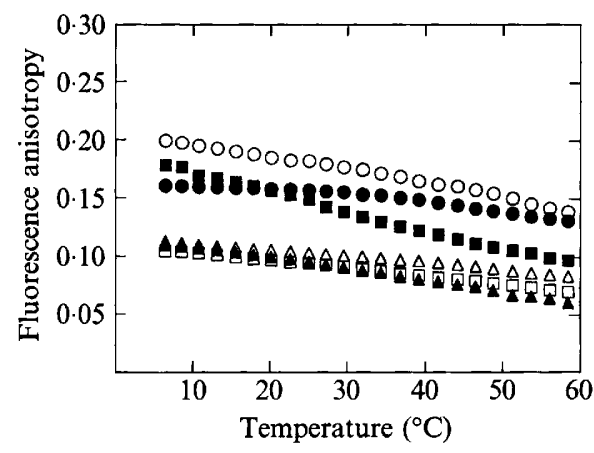

Fig. 2. Temperature dependence of steady-state fluorescence anisotropy in DPH-labelled liposomes derived from cellular phospholipids of $E$. chevalieri grown at various $a_{\mathrm{w}} . \mathbf{U}, 0.995 a_{\mathrm{w}} ; \square, 0.97 a_{\mathrm{w}} ; \boldsymbol{\Lambda}, 0.93 a_{\mathrm{w}}$; $\triangle, 0.90 a_{\mathrm{w}} ; 0,0.86 a_{\mathrm{w}} ; 0,0.80 a_{\mathrm{w}}$

a continuous decrease, with no marked discontinuity or inflection in slope, and consequently no liquid-crystalline to gel phase transition temperature in phospholipid domains could be visualized. The temperature dependence of the structural order parameter of DPH in the various samples of liposomes was also investigated (Figs 3-5). Discrete discontinuities in slope were discernible in plots of $S$ vs temperature. Corresponding slopes for the $S$ data (smoothed first derivatives) vs temperature were plotted to confirm the existence and the location of breaks in the temperature profiles of $S$ (Lynch et al., 1987). Each liposomal membrane exhibited characteristic break points. For liposomal membranes obtained at $0.995 a_{\mathrm{w}}$, three successive breaks were visualized in the derivative plot of $S$ (Fig. $3 b$ ). With 0.97 and $0.93 a_{\text {w }}$ liposomal membranes, two breaks were found at 27 and $44^{\circ} \mathrm{C}$ (Fig. $3 d$ ) and 20 and $44^{\circ} \mathrm{C}$ (Fig. $4 b$ ), respectively. For liposomal membranes from mycelia obtained at lower $a_{\mathrm{w}}$ (Figs 4 and 5), derivative plots exhibited continuous changes in slope, from 32 to $46^{\circ} \mathrm{C}$ at $0.90 a_{\mathrm{w}}$, from 22 to $44{ }^{\circ} \mathrm{C}$ at $0.86 a_{\mathrm{w}}$, and from 22 to $50^{\circ} \mathrm{C}$ at $0.80 a_{\mathrm{w}}$. The mid-points of the inflections in the derivative

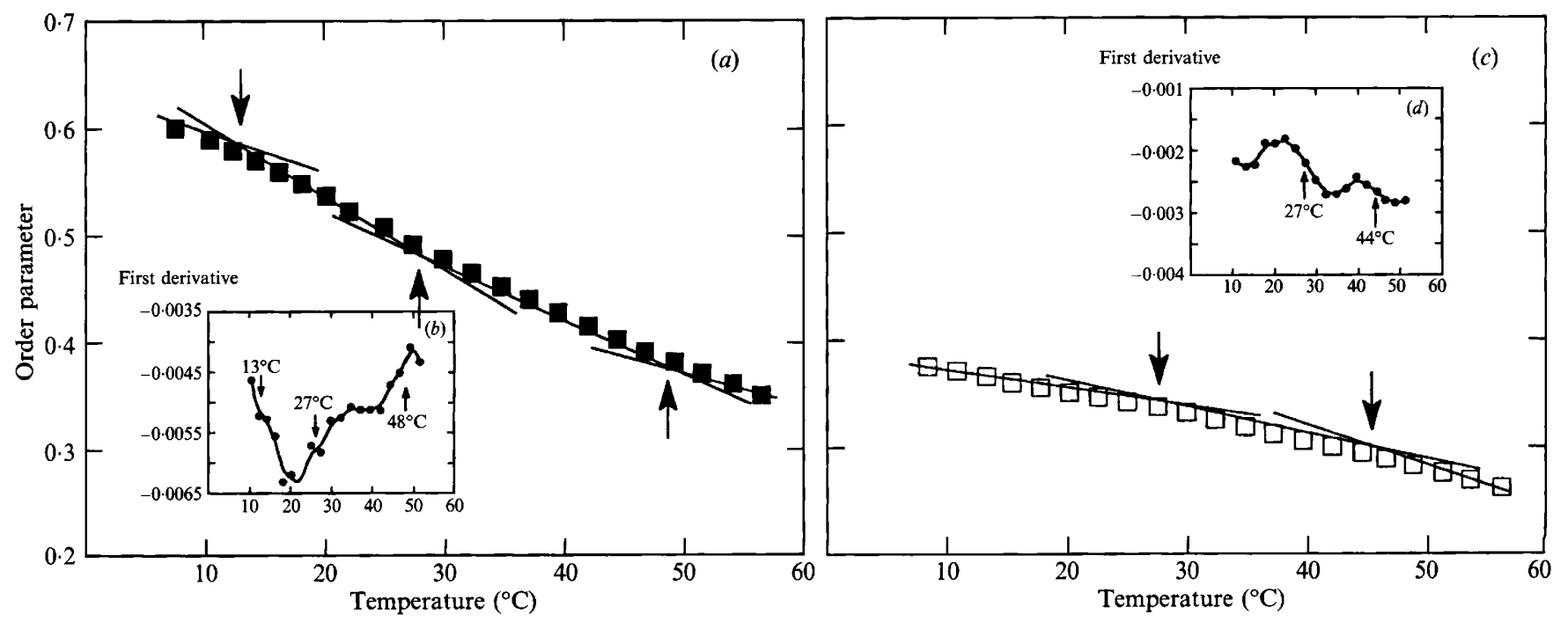

Fig. 3. Temperature dependence of the order parameter of DPH in liposomal membranes derived from cellular phospholipids of $E$. chevalieri grown at $0.995 a_{\mathrm{w}}(a)$ and $0.97 a_{\mathrm{w}}(c)$. The corresponding derivatives were calculated, smoothed and plotted vs temperature in $(b)$ and $(d)$ for 0.995 and $0.97 a_{\mathrm{w}}$, respectively. Break points are indicated by arrows. 

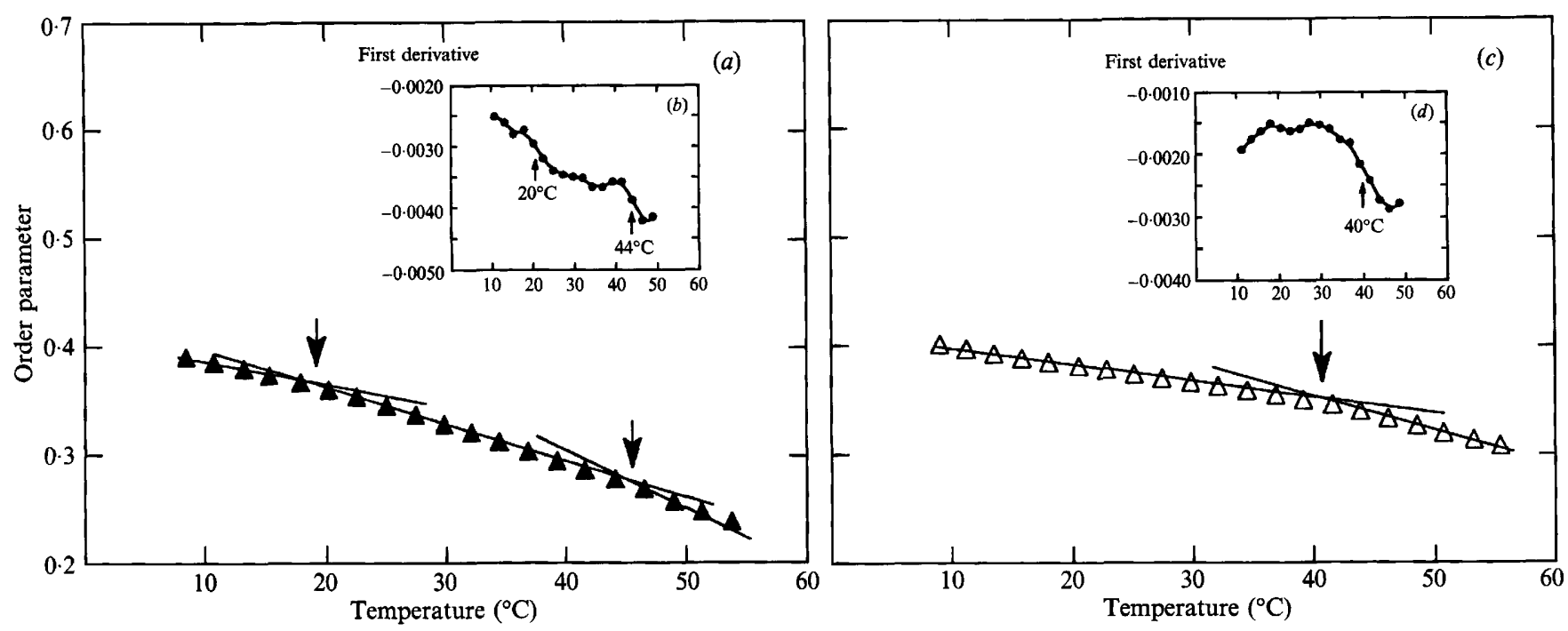

Fig. 4. Temperature dependence of the order parameter of DPH in liposomal membranes derived from cellular phospholipids of $E$. chevalieri grown at $0.93 a_{\mathrm{w}}(a)$ and $0.90 a_{\mathrm{w}}(c)$. The corresponding derivatives were calculated, smoothed and plotted vs temperature in $(b)$ and $(d)$ at 0.93 and $0.90 a_{\mathrm{w}}$, respectively. Break points are indicated by arrows.
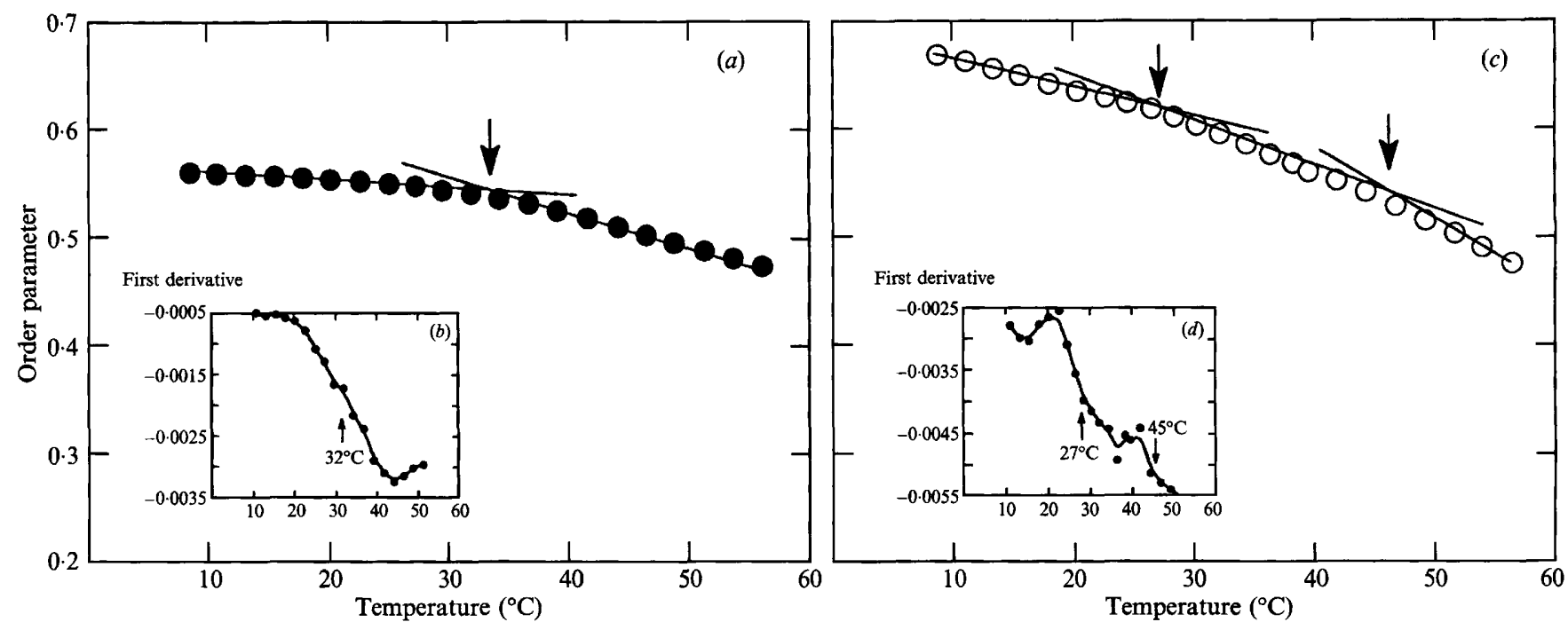

Fig. 5. Temperature dependence of the order parameter of DPH in liposomal membranes derived from cellular phospholipids of $E$. chevalieri grown at $0.86 a_{\mathrm{w}}(a)$ and $0.80 a_{\mathrm{w}}(c)$. The corresponding derivatives were calculated, smoothed and plotted vs temperature in $(b)$ and $(d)$ at 0.86 and $0.80 a_{\mathrm{w}}$, respectively. Break points are indicated by arrows.

plots coincided with discontinuities visualized in the temperature profile of $S$, with values of 40,32 and $27^{\circ} \mathrm{C}-45^{\circ} \mathrm{C}$, at $0.90,0.86$ and $0.80 a_{\mathrm{w}}$, respectively.

\section{Lifetime and rotational relaxation time}

DPH lifetime and calculated rotational correlation time at $25^{\circ} \mathrm{C}$ in liposome membranes derived from $E$. chevalieri grown at various $a_{\mathrm{w}}$ are shown in Table 2 .
Considering the standard deviations, the data for DPH lifetime and rotational correlation time were not significantly different for any of the analysed samples.

\section{Discussion}

This work confirms the considerable ability of the fungus $E$. chevalieri to grow at low $a_{\mathrm{w}}$. The optimum for mycelial growth at $a_{\mathrm{w}} 0.90$ and the restricted growth at very high 
$a_{\mathrm{w}}\left(0.995 a_{\mathrm{w}}\right)$ highlighted its xerophilic behaviour (Fig. 1). Studying the germination and growth of xerophilic fungi, Pitt and Hocking (1977) found that $0.94 a_{\mathrm{w}}$ was the optimum for the growth of $E$. chevalieri. Differences in conditions of culture and growth measurements may explain this variation between the two studies.

In agreement with the data on Ascomycetes published by Wassef (1977), the fatty acid composition of $E$. chevalieri phospholipids showed a preponderance of fatty acids with chain lengths of 16 and 18 carbon atoms. Changes in $a_{\mathrm{w}}$ of the growth medium affected the fatty acid composition of these phospholipids, causing a decrease in $\mathrm{C}_{18: 2}$ and an increase in $\mathrm{C}_{18: 1}$. Thus, one effect of growth of $E$. chevalieri at low $a_{\mathrm{w}}$ was a marked increase in the level of phospholipid fatty acid saturation as seen both by the decrease in unsaturation index and in the $\mathrm{C}_{18: 2} / \mathrm{C}_{18: 1}$ ratio (Table 1 ). Modifications of fatty acid composition due to decreased $a_{\mathrm{w}}$ of the growth medium have been observed for other fungal systems. TunbladJohansson \& Adler (1987) observed that increased salinity produced only minor changes in the fatty acid composition of phospholipids of the non-tolerant Saccharomyces cerevisiae species, whereas a marked decrease in $\mathrm{C}_{18}$ polyenic acids with a concomitant increase in $\mathrm{C}_{18: 1}$ was shown in the phospholipids of the osmotolerant yeast. Hosono (1992) described a decrease in $\mathrm{C}_{18: 2}$ and a corresponding increase in $\mathrm{C}_{18: 1}$ when the salt-tolerant yeast $Z$ ygosaccharomyces rouxii was grown in medium containing $15 \%(\mathrm{w} / \mathrm{v}) \mathrm{NaCl}$. Saad (1992) reported the accumulation of phospholipids and saturated fatty acids at low $a_{\mathrm{w}}$. Such modifications of saturation of phospholipid fatty acids could be sufficient to affect the membrane permeability and explain, at least partially, cellular acclimation to decreased $a_{\mathrm{w}}$. Indeed, xerophilic fungi have been reported to accumulate osmoregulator solutes (i.e. glycerol) in response to reduced $a_{\mathrm{w}}$ while the nontolerant species leaked significant amounts of glycerol to the surrounding medium (Brown, 1978; Luard, 1982; Hocking, 1986, 1988). The response of the tolerant species to decreasing $a_{\mathrm{w}}$ is at the level of glycerol permeation and transport, whereas that of the nontolerant one is only metabolic (Hocking, 1988).

The changes observed in the saturation of phospholipid $\mathrm{C}_{18}$ fatty acids were expected to cause an increase in the membrane packing as indicated by Brenner (1984) and consequently to reduce the membrane permeability to osmoregulator solutes. More information about the membrane physical properties of $E$. chevalieri cultivated at reduced $a_{\mathrm{w}}$ was obtained from measurements of steady state fluorescence anisotropy $\left(r_{\mathrm{s}}\right)$ in DPH-labelled liposome systems prepared from cellular phospholipids of E. chevalieri. The lipid order parameter $(S)$, determining the degree to which fluorophore movement in the membrane is restricted by the molecular packing of surrounding lipids (Van Blitterswijk et al., 1981; Van der Meer et al., 1986), was calculated from $r_{\mathrm{s}}$ data.

Except at $0.995 a_{\mathrm{w}}$, the decreasing $a_{\mathrm{w}}$ values for mycelial growth were accompanied by increasing $r_{\mathrm{s}}$ and $S$ values (Table 2). This result indicates that at lower $a_{\mathrm{w}}$ growth values $E$. chevalieri produces membranes in which the movements of lipid acyl chains are restricted. However, the $r_{\mathrm{s}}$ and $S$ values never reached those of highly structurally ordered (rigid) membranes such as DPH-labelled liposomes of dipalmitoyl-phosphatidylcholine (gel phase) or eye lens membranes which at $25^{\circ} \mathrm{C}$, have values of 0.31 and 0.87 for $r_{\mathrm{s}}$ and $S$, respectively (Van Blitterswijk et al., 1981). Values were rather close to these of fluid biological membranes such as leukaemic cell membranes. Consequently, liposomal membranes prepared from cellular phospholipids of $E$. chevalieri at $25^{\circ} \mathrm{C}$ would be in the fluid liquid-crystalline state, whatever the $a_{\mathrm{w}}$ for growth. The rotational correlation time $(\rho)$ values calculated from $\tau$ and $r_{s}$ data of excited probe (DPH) (Table 2) indicated no significant variation as a function of $a_{\mathrm{w}}$ and allowed us to confirm that the static component $r_{\infty}$ (proportional to the order parameter $S$ ) prevailed in the interpretation of $r_{\mathrm{s}}$ changes in our experiments (see Methods). Under these conditions, membrane fluidity, a parameter which describes membrane dynamics (Van Ginkel et al., 1989) would be constant in our experiments, while the order parameter increased largely when $a_{\mathrm{w}}$ decreased.

This rigidification of the fungal membrane observed at low $a_{\mathrm{w}}$ is consistent with the increase in the level of phospholipid fatty acid saturation described previously. Similarly, Hosono (1992) reported increased rigidity of the plasma membrane (on the basis of fluorescence polarization values) associated with an increase in saturation of phospholipid fatty acids when the salttolerant yeast $Z$ ygosaccharomyces rouxii was grown under high salinity conditions.

By plotting the order parameter $(S)$ and its first derivative as a function of temperature, phase transitions of liposomes prepared from cellular phospholipids of $E$. chevalieri grown at different $a_{\mathrm{w}}$ were visualized. Many break points, defined from inflections in slope and indicative of phase transitions, were observed overall in the temperature range $20-48{ }^{\circ} \mathrm{C}$. These profiles were certainly due to the complex composition of phospholipids constituting the liposomes prepared at different $a_{\mathrm{w}}$. Mixed lipid systems such as those found in cell membranes do not exhibit a well-defined transition from gel to liquid-crystalline state. Rather, chain melting occurs over a broad temperature range during which phase segregations of lipids occur (Chapman, 1975). Changes in slope of plots such as those observed in Figs 3-5 may indicate the start and end points of these 
processes. However, chain melting seems to shift to higher temperatures with decreasing $a_{\mathrm{w}}$, especially between 0.97 and $0.90 a_{\mathrm{w}}$. This observation is in agreement with Yamazaki et al. (1992), who reported an increase in phase transition temperatures of vesicles of dipalmitoylphosphatidylcholine associated with higher ethylene glycol concentrations.

As transition temperatures depend not only on the fatty acid composition of phospholipids but also on the polar head group (Brenner, 1984), further investigations are needed to determine the composition of different classes of phospholipids present in membranes of $E$. chevalieri grown at various $a_{\mathrm{w}}$. It would be necessary to estimate the contribution of sterols and membrane proteins (especially in plasma membrane) to membrane physical properties by comparing DPH steady-state fluorescence anisotropy in isolated native membranes with that in liposomes prepared from membrane lipid extracts.

In conclusion, our results reveal that changes in water activity of growth leads to modifications in the phospholipid fatty acid saturation and in the membrane molecular order. These changes in the membrane structure are an adaptive response of the fungus to water stress, which could explain the differences of permeabilities observed for osmoregulator solutes in tolerant and non-tolerant species.

\section{References}

AdLER, L. \& LiLJENBERG, C. (1981). Sterol content, fatty acid composition of phospholipids, and permeability of labeled ethylene glycols in relation to salt-tolerance of yeasts. Physiologia Plantarum 53, 368-374.

BARTLETT, G. R. (1959). Phosphorus assay in column chromatography. Journal of Biological Chemistry 234, 466-468.

Biondi, A. C., Feliz, M. R. \& Disalvo, E. A. (1991). Surface changes induced by osmotic stress and its influence on the glycerol permeability in lipid bilayers. Biochimica et Biophysica Acta 1069, 5-13.

Bizot, H., Tome, D., Drapron, R., Gullbot, A. \& Multon, J. L. (1978). Les aliments à humidité intermédiaire. In Série Synthèse bibliographique, no. 16. Paris: CDIUPA et APRIA.

BLIGH, E. G. \& DYer, W. J. (1959). A rapid method of total lipid extraction and purification. Canadian Journal of Biochemistry and Physiology 37, 911-917.

BRENNER, R. R. (1984). Effect of unsaturated acids on membrane structure and enzyme kinetics. Progress in Lipid Research 23, 69-96.

Brown, A. D. (1978). Compatible solutes and extreme water stress in eukaryotic micro-organisms. Advances in Microbial Physiology 17, 181-242.

Chapman, D. (1975). Phase transitions and fluidity characteristics of lipids and cell membranes. Quarterly Reviews of Biophysics 8, 185-235.

CHEN, C. S. (1987). Sorption isotherm and freezing point depression equation of glycerol solutions. American Society of Agricultural Engineers 30, 279-282.

Christie, W. W. (1982). Lipid Analysis. Oxford: Pergamon Press.

Folch, J., LeEs, M. \& Sloane-Stanley, G. H. (1957). A simple method for the isolation and purification of total lipids from animal tissues. Journal of Biological Chemistry 226, 497-509.
Hazel, J. R. \& Williams, E. (1990). The role of alterations in membrane lipid composition in enabling physiological adaptation of organisms to their physical environment. Progress in Lipid Research 29, 167-227.

HEYN, M. P. (1979). Determination of lipid order parameters and rotational correlation times from fluorescence depolarization experiments. FEBS Letters 108, 359-364.

HockING, A. D. (1986). Effect of water activity and culture age on the glycerol accumulation patterns of five fungi. Journal of General Microbiology 132, 269-275.

HockING, A. D. (1988). Strategies for microbial growth at reduced water activities. Microbiological Sciences 5, 280-284.

Hosono, K. (1992). Effect of salt stress on lipid composition and membrane fluidity of the salt-tolerant yeast Zygosaccharomyces rouxii. Journal of General Microbiology 138, 91-96.

KATES, M. \& HAGEN, P. O. (1964). Influence of temperature on fatty acid composition of psychrophilic and mesophilic Serratia species. Canadian Journal of Biochemistry 42, 481-488.

LACEY, J. (1989). Pre- and post-harvest ecology of fungi causing spoilage of foods and other stored products. Journal of Applied Bacteriology Symposium Supplement 67, 115-255.

Lakowicz, J. R. (1983). Principles of Fluorescence Spectroscopy. New York: Plenum Press.

LENTZ, B. R. (1989). Membrane 'fluidity' as detected by diphenylhexatriene probes. Chemistry and Physics of Lipids 50, $171-190$.

LENTZ, B. R., MOORE, B. M. \& BARRow, D. A. (1979). Light-scattering effects in the measurement of membrane microviscosity with diphenylhexatriene. Biophysical Journal 25, 489-494.

LUARD, E. J. (1982). Accumulation of intracellular solutes by two filamentous fungi in response to growth at low steady state osmotic potential. Journal of General Microbiology 128, 2563-2574.

LYNCH, D. V., LEPOCK, J. R. \& THOMPSON, J. E. (1987). Temperatureinduced changes in lipid fluidity alter the conformation of proteins in senescing plant membranes. Plant Cell Physiology 28, 787-797.

MARANGONI, A. G. (1992). Steady-state fluorescence polarization spectroscopy as a tool to determine microviscosity and structural order in food systems. Food Research International 25, 67-80.

MoRrison, W. R. \& SMITH, L. M. (1964). Preparation of fatty acid methyl esters and dimethylacetals from lipids with boron fluoridemethanol. Journal of Lipid Research 5, 600-608.

PITT, J. I. (1975). Xerophilic fungi and the spoilage of foods of plant origin. In Water Relations of Foods, pp. 273-307. Edited by R. B. Duckworth. London: Academic Press.

PiTT, J. I. \& HockING, A. D. (1977). Influence of solute and hydrogen ion concentration on the water relations of some xerophilic fungi. Journal of General Microbiology 101, 35-40.

RAPER, K. B. \& FenNell, O. (1965). The Genus Aspergillus. Baltimore: Williams and Wilkins.

Richard-Molard, D., Lesage, L. \& CaHAGNiER, B. (1985). Effect of water activity on mold growth and mycotoxin production. In Properties of Water in Foods, pp. 273-292. Edited by D. Simatos \& J. L. Multon. Dordrecht, The Netherlands: Martinus Nijhoff.

Russell, N. J. (1989). Functions of lipids: structural roles and membrane functions. In Microbial Lipids, Vol. 2, pp. 279-365. Edited by C. Ratledge \& S. G. Wilkinson. London: Academic Press.

SAAD, R. R. (1992). Effect of water activity on growth and lipids of xerophilic fungi, Aspergillus repens and Aspergillus amstelodami. Zentralblatt für Mikrobiologie 147, 61-64.

SHINITZKY, M. \& BARENHOLZ, Y. (1978). Fluidity parameters of lipid regions determined by fluorescence polarization. Biochimica et Biophysica Acta 515, 367-394.

SHINITZKY, M. \& INBAR, M. (1976). Microviscosity parameters and protein mobility in biological membranes. Biochimica et Biophysica Acta 433, 133-149.

Singer, S. J. \& Nicolson, G. L. (1972). The fluid mosaic model of the structure of cell membranes. Science 175, 720-731.

TUNBLAD-JohansSON, I. \& ADLER, L. (1987). Effects of sodium chloride concentration on phospholipid fatty acid composition of yeasts differing in osmotolerance. FEMS Microbiology Letters 43, 275-278.

Van BlitterswiJk, W. J., Van Hoeven, R. P. \& Van Der Meer, B. W. (1981). Lipid structural order parameters (reciprocal of fluidity) in 
biomembranes derived from steady-state fluorescence polarization measurements. Biochimica et Biophysica Acta 644, 323-332.

Van Der Meer, B. W., Van Hoeven, R. P. \& Van BlitterswiJk, W. J. (1986). Steady-state fluorescence polarization data in membranes. Resolution into physical parameters by an extended Perrin equation for restricted rotation of fluorophores. Biochimica et Biophysica Acta 854, 38-44.

Van Ginkel, G., Van langen, H. \& LeVine, Y. K. (1989). The membrane fluidity concept revisited by polarized fluorescence spectroscopy on different model membranes containing unsaturated lipids and sterols. Biochimie 71, 23-32.

Wassef, M. K. (1977). Fungal lipids. Advances in Lipid Research 15, 159-232.

Yamazaki, M., Ohshika, N., Kashiwagi, N. \& Asano, T. (1992) Phase transitions of phospholipid vesicles under osmotic stress and in presence of ethylene glycol. Biophysical Chemistry 43, 29-37. 